\section{§33. One Dimensional Simulation on Stability of Detached Plasma in a Tokamak Divertor}

Nakazawa, S., Nakajima, N., Okamoto, M., Ohyabu, N.

One of the most severe problems of the nextgeneration fusion reactors is that of heat load onto the divertor target. A solution for reducing the heat load is to produce the 'detached plasma' in the divertor region. The tokamak divertor experiments, however, show that the location of detachment front (or radiation front) is unstable: the radiation front moves inside the LCFS, and consequently it cools the core plasma. [1] Therefore, the stability of such a detached configuration is an important problem.

In order to investigate the properties of the detachment front, we have carried out numerical simulations using one-dimensional fluid code. In particular, this simulation study is focused on the problem of whether the location of detachment front is stable or not.

We consider the hydrogen plasma confined in a SOL region between the midplane and the divertor target. The plasma fluid is described with the time-dependent transport equations based on the single-fluid theory, where ionization, charge exchange, radiative and threebody recombination effects are taken into account. They are solved in the direction parallel to a magnetic field line. The neutral gas is approximately treated by using a diffusion model. Carbon is the only impurity species considered. The fixed fraction model and the coronal approximation are used to give the impurity density and radiation loss, respectively.

We observed how the SOL plasma changes from the attached states to the detached states as the heat externally injected into the system $W_{\text {in }}$ is decreased. The main results are as follows. (1) Stable detached solutions exist, where the temperature at the divertor target is $T_{d} \sim 2 \mathrm{eV}$, and the heat flux onto the target $\gamma n T v$ is significantly decreased to around $10 \%$ of the injection power $W_{i n}$ (Fig. 1). (2) Whenever such stable detached states are attained, the strong radiation front is in contact with or at a small distance from the divertor target. Probably, such a condition will not be favorable, because the target will be subjected to an amount of radiation. (3) When the input power is decreased below a critical value in magnitude, the radiation front moves towards the X-point, cooling the SOL plasma (Fig. 2). For such cases, no staionary solutions are observed. These results implicitly indicate that it is not easy to use a detached plasma from the view point of the location-stability of detachment front.
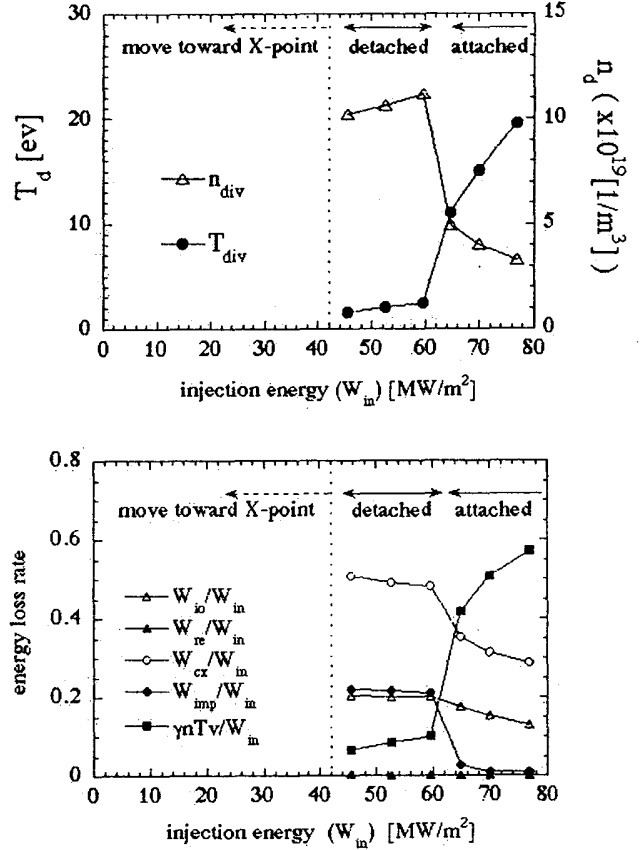

Figure 1: Injection-power dependences.
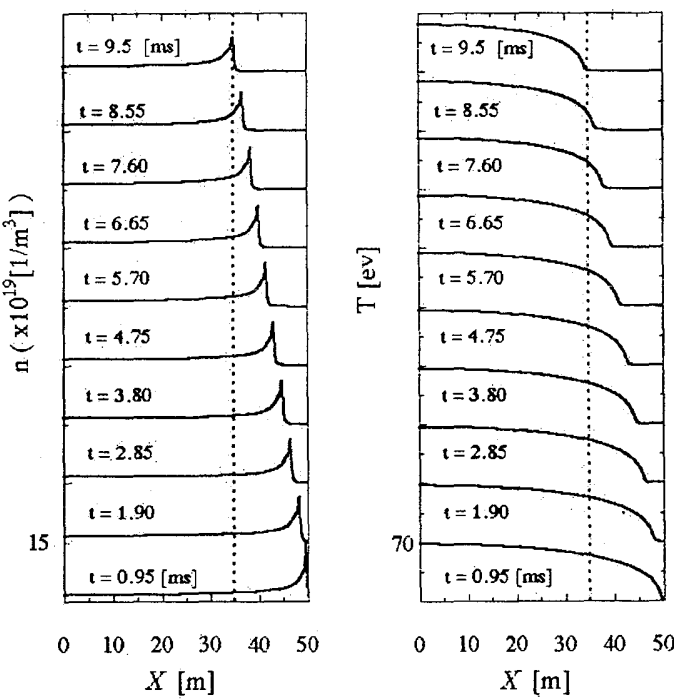

Figure 2: Profiles of $n$ and $T$ at various times. Vertical dotted line represents the $x$ position of $\mathrm{X}$-point.

\section{References}

[1] J. A. Goetz, et al., Phys. Plasmas $\underline{3}$ (5), (1996) 1908 